\title{
Highly sensitive, reproducible and uniform SERS substrates with a high density of three-dimensionally distributed hotspots: gyroid-structured Au periodic metallic materials
}

\author{
Liping $\mathrm{Wu}^{1,3}$, Wanlin Wang ${ }^{2,3}$, Wang Zhang ${ }^{1}$, Huilan $\mathrm{Su}^{1}$, Qinglei Liu ${ }^{1}$, Jiajun $\mathrm{Gu}^{1}$, Tao Deng ${ }^{1}$ and Di Zhang ${ }^{1}$
}

Surface-enhanced Raman spectroscopy (SERS) is an important tool for the analytical, trace detection of many inorganic and organic materials, especially for materials involved in medical care, food safety and environmental pollution. Numerous efforts have been dedicated to exploring periodic metallic materials with a high density of hotspots. However, for most periodic metallic materials fabricated by top-down and bottom-up approaches, the distribution of hotspots is restricted to one or two dimensions. Here, for the first time, we report the successful fabrication of a bio-inspired bicontinuous gyroid-structured Au SERS substrate with a high density of three-dimensionally (3D) distributed hotspots. The as-required gyroid-structured substrates were demonstrated to be highly sensitive, reproducible and uniform, with an enhancement factor of up to $10^{9}$. Finite-difference time domain (FDTD) simulations were conducted to reveal the mechanism leading to the high enhancement and we found that the interconnected helices in the gyroid structure not only increase the hotspot density but also contribute to increasing the scattering cross-section of the incident laser. The substrate was then adopted for the SERS detection of bis(2-ethylhexyl) phthalate, the most frequently used plasticizer in food, paints, house-hold items, perfumes and so on, and reached a detection limit of $1 \mathrm{fM}$, which is among the best results ever reported. Moreover, the mechanism deduced here will provide insight into the future design and selection of novel surface plasmonic resonance substrates, as many other bicontinuous interconnected systems are available.

NPG Asia Materials (2018) 10, e462; doi:10.1038/am.2017.230; published online 12 January 2018

\section{INTRODUCTION}

The fabrication of surface plasmonic resonance (SPR) materials with ultra-high plasmonic enhancement has long been a critical research area due to their broad applications as chemical sensors, biological sensors, plasmonic solar cells, photocatalysts and other environmentally friendly devices. ${ }^{1-3}$ Among these applications, the most famous is the surface-enhanced Raman spectroscopy (SERS) detection of trace analytes, especially for analytes involved in medical care, food safety and environmental pollution. Various nanoparticle systems with novel nanomorphologies and their assemblies have been fabricated and reported to show excellent SPR performance. ${ }^{2-5}$ However, SERS nanostructures composed of nanoparticles face significant challenges in achieving a reproducible and uniform Raman response due to the difficulty in fabricating uniform SERS active sites. Periodic metallic materials could provide hotspots with high density, reproducibility and uniformity, which completely meet the requirement of valid SERS substrates, and therefore numerous efforts have been dedicated to engineering periodic metallic materials with novel nanomorphologies, including nanofingers, ${ }^{6}$ nanorods, ${ }^{7}$ nanoridges ${ }^{8}$ and other hierarchical structures. $^{9-11}$ However, apart from their high cost and timeconsuming synthesis, most of these substrates suffer from a limited density of hotspots distributed in either one-dimensional or twodimensional space.

In recent years, as one of the most stable bicontinuous structures, the gyroid structure has attracted great attention due to its unique structural properties. ${ }^{12}$ It adopts a body centered cubic bcc lattice with triply periodic symmetry $\left(I 4_{1} 32\right)$ and intrinsic chirality. ${ }^{13}$ Most importantly, the interconnected helices enable us to engineer the distribution of hotspots in all three dimensions. ${ }^{14}$ Gyroid-structured motifs self-assembled from block polymer systems have frequently been adopted as hard templates to fabricate Au plasmonic materials and the optical properties of such systems have been thoroughly investigated. $^{4,15-21}$ Three-dimensional (3D) gyroid-structured Au nanoparticles with a unit cell size below $100 \mathrm{~nm}$ have been reported to show excellent SPR performance originating from the abundant exposed nanotips on the external surface. ${ }^{15,17}$ However, the hotspots

\footnotetext{
${ }^{1}$ School of Materials Science and Engineering, State Key Lab of Metal Matrix Composite, Shanghai Jiao Tong University, Shanghai, China and ${ }^{2}$ College of Electronic Science and Technology, Shenzhen University, Shenzhen, China

${ }^{3}$ These authors contributed equally to this work.

Correspondence: Dr W Zhang or Professor D Zhang, State Key Lab of Metal Matrix Composite, Shanghai Jiao Tong University, 800 Dongchuan Road, Shanghai 200240 , China. E-mail: zhangdi@sjtu.edu.cn or wangzhang@sjtu.edu.cn

Received 16 July 2017; revised 13 October 2017; accepted 6 November 2017
} 


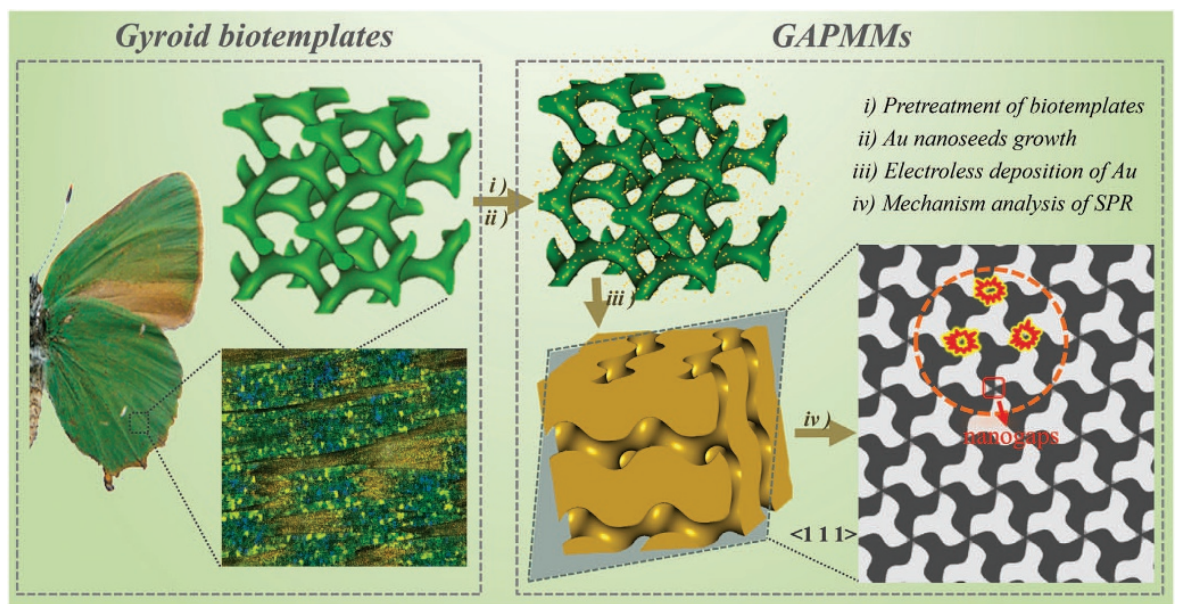

Figure 1 Schematic illustration of the fabrication of GAPMMs together with the analysis of mechanism of their ultrahigh SPR efficiency.

were still confined to a two-dimensional distribution of limited density and enhancement, and the merits of the gyroid morphology remain unexploited.

Engineering the hotspot distribution inside periodic metallic materials usually requires precise control of the volume fraction (VF) of the metals. ${ }^{22,23}$ However, bottom-up gyroid templates from self-assembly systems are usually adopted as hard templates and are not feasible for continuous regulation of the VF. In our previous work, we proved that biotemplates, such as butterfly wings, can be used as soft templates after pretreatment and are versatile for the electroless deposition of metal films. ${ }^{24}$ Here we use gyroid-structured photonic crystals from the wing scales of the butterfly Callophrys rubi as soft templates after activating the biomaterials on the external surface. A series of gyroid-structured Au periodic metallic materials (GAPMMs) were fabricated by properly controlling the deposition time $\left(t_{\mathrm{d}}\right)$ using an electroless deposition method, as shown in Figure 1. Quantitative analysis revealed that the density of the 3D-distributed nanogaps and the SPR enhancement of the GAPMMs are the highest at a $t_{\mathrm{d}}$ of $4 \mathrm{~h}$, among other $t_{\mathrm{d}}$ varying from 1 to $6 \mathrm{~h}$. Further finite-difference time domain (FDTD) simulations confirm that apart from the high density of 3D-distributed hotspots, the bicontinuous interconnected helices greatly increase the scattering cross-section, thereby increasing the SPR enhancement. The as-acquired GAPMMs demonstrated ultra-high SPR enhancement (an enhancement factor of $0.8 \times 10^{9}$ ) with superior reproducibility, uniformity and stability. For crystal violet (CV) molecules, the SERS detection limit of GAPMMs with a $t_{\mathrm{d}}$ of $4 \mathrm{~h}$ (GAPMMs-4h) reached $10^{-12} \mathrm{M}$, which is four orders of magnitude lower than that of the gyroid-structured $\mathrm{Au}$ plasmonic materials templated from block polymer systems ${ }^{15}$ and two orders of magnitude lower than that of the commercial SERS substrates Q-SERS; however, the cost is far less than that of Q-SERS. Meanwhile, the detect limit for bis(2-ethylhexyl) phthalate (DEHP) molecules, the most frequently used plasticizer in food, paints, house-hold items, perfumes and so on, reached $1 \mathrm{fM}$, which is among the best results ever reported. Moreover, the mechanism deduced here will provide insight into the future design and selection of novel SPR substrates, as many other bicontinuous interconnected systems are available. ${ }^{25-27}$

\section{EXPERIMENTAL PROCEDURES}

\section{Fabrication of GAPMMs}

The fabrication of GAPMMs using a biotemplate involves three steps as follows: (1) amination of the biotemplate: butterfly wings were first immersed in ethanol for 30 min to preliminarily clean the surface of the wings. Afterwards, the wings were aminated by immersion in dilute 8 vol\% nitric acid for $2 \mathrm{~h}$ and then in an ethanol solution of ethanediamine ( $25 \mathrm{vol} \%$ ) for $6 \mathrm{~h}$. (2) Nanoseed growth: the aminated wings were immersed for $4 \mathrm{~h}$ in a mixed solution of $\mathrm{HAuCl}_{4}(0.2 \mathrm{wt}$ $\%$ ), which was prepared by dissolving $\mathrm{HAuCl}_{4}$ in equal weights of ethanol and deionized water. Afterwards, the wings were removed, rinsed with deionized water and immersed in a $0.1 \mathrm{M} \mathrm{NaBH}_{4}$ aqueous solution for $2 \mathrm{~min}$ to form a homogeneous layer of $\mathrm{Au}$ nanoseeds on the activated biosurface by reducing the adsorbent $\mathrm{AuCl}_{4}{ }^{-}$on the aminated biosurface. (3) Au deposition: the Au-nanoseedfunctionalized butterfly wings were immersed in a specific electroless deposition solution for a given time. The electroless deposition solution was prepared by mixing solutions $\mathrm{A}$ and $\mathrm{B}$ in equal volume (A: $2 \mathrm{~g} \mathrm{NaCl}, 1 \mathrm{~g} \mathrm{HAuCl}_{4}, 100 \mathrm{ml}$ deionized water; B: $2 \mathrm{~g}$ diethanolamine (DEA), $20 \mathrm{ml}$ ethanol, $100 \mathrm{ml}$ deionized water). Both solutions A and $\mathrm{B}$ were prepared and stored at $4{ }^{\circ} \mathrm{C}$ before use. All experiments were conducted at room temperature.

\section{Characterization of the GAPMMs}

Scanning electron microscopy (SEM) was performed using a fieldemission SEM instrument (Quanta 250 from FEI, $20 \mathrm{kV}$, Hillsboro, OR, USA) equipped with energy-dispersive X-ray spectroscopy analysis capability (Oxford Instruments, Oxford city, UK, $80 \mathrm{~mm}^{2}$ detector). Transmission electron microscopy (TEM) images of the cross-section of the scales were acquired using a Bio-TEM FEI Tecnai Spirit Biotwin. X-ray diffraction measurements were conducted using a D/max-2550 instrument (CuK $\alpha$, Rigaku Corporation, Tokyo, Japan). The extinction spectrum of the Au gyroid scales was acquired using a Microscope Systems ARM61 manufactured by Shanghai Ideaoptics Corporation, Shanghai, China.

\section{TEM projections}

TEM cross-sections of the samples were prepared by sequentially immersing the samples in a mixture of acetone and phenolic resin in ratios of 2:1, 1:1 and 1:2 for $1 \mathrm{~h}$, in order at room temperature. Then, the samples were transferred into the phenolic resin and stored for polymerization in a drying oven at $60^{\circ} \mathrm{C}$ for $48 \mathrm{~h}$. Slices of the scale cross-sections were obtained using a frozen ultra-thin slicing machine (Leica ULTRACUT UC6, Leica Microsystems Inc., Wetzlar, Germany), which were then transferred onto copper grids for observation. The simulated TEM projections of different plane directions were 


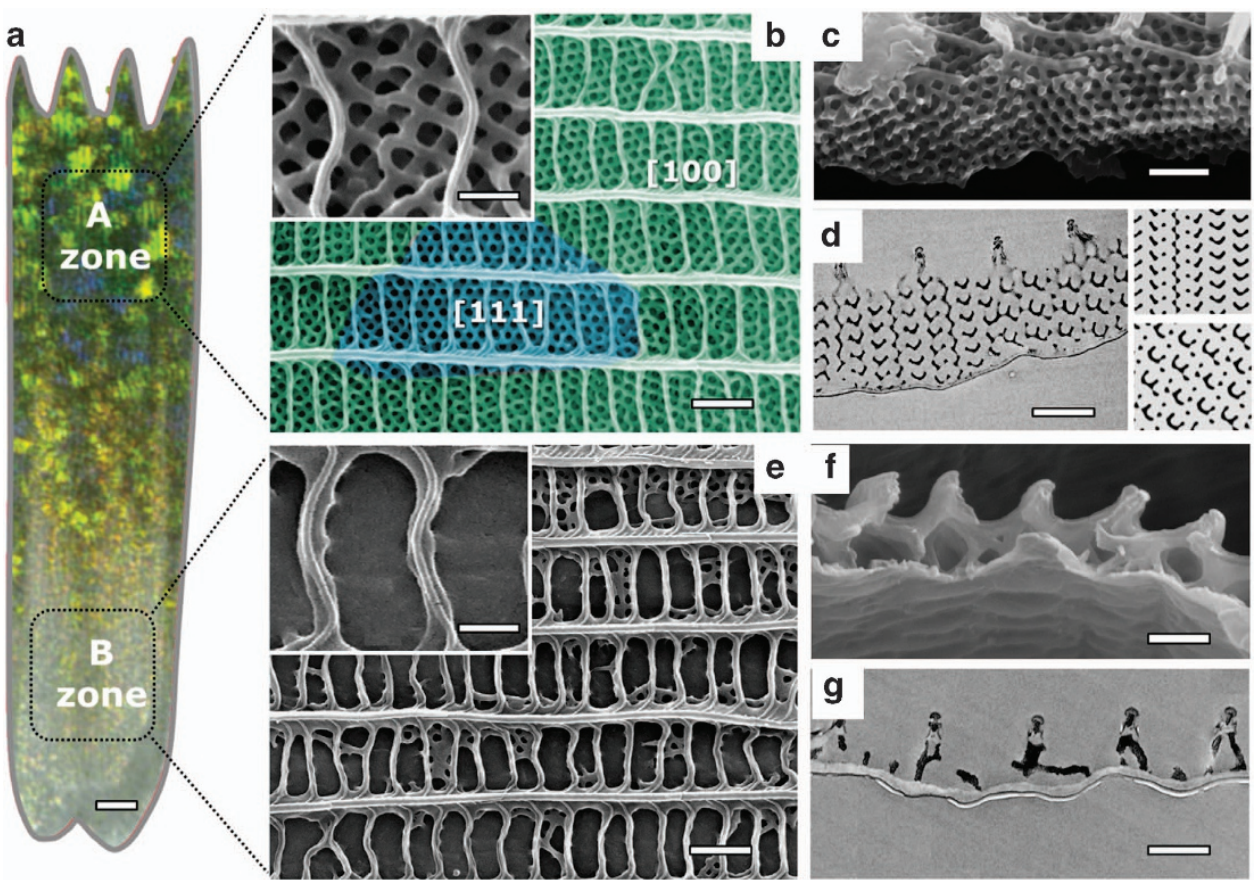

Figure 2 Optical, SEM and TEM characterizations of the gyroid structure in the butterfly wing scales. (a) Optical image of a scale with gyroid structure from butterfly $C$. rubi. (b) SEM image of zone A. Inset is a zoomed-in view of the $\left[\begin{array}{lll}1 & 0 & 0\end{array}\right]$ zone. (c, d) SEM and TEM images of the cross-section of the G scale in

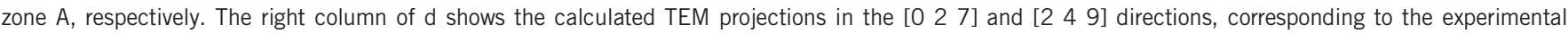
images $(t=1$, equation S1). (e) SEM image of zone B. Inset is a zoomed-in view. (f, $\mathbf{g})$ SEM and TEM images of the cross-section of the G scale in zone B, respectively. Scale bars: (a) $5 \mu \mathrm{m}$; (b-g) $1 \mu \mathrm{m}$; insets of $\mathbf{b}$ and $\mathbf{e : ~} 0.5 \mu \mathrm{m}$.

modeled by MATLAB 2010 Mathworks Inc., Natick, MA, USA using the following equations ${ }^{4}$ :

Gyroid:

$$
\begin{aligned}
f(x, y, z) & =\sin x \cos y+\sin y \cos z+\sin z \cos x \\
& =\mathrm{t}(-1.5 \leq \mathrm{t} \leq 1.5)
\end{aligned}
$$

Double gyroid:

$$
\begin{aligned}
f(x, y, z) & =\sin x \cos y+\sin y \cos z+\sin z \cos x \\
& = \pm \mathrm{t}(-1.5 \leq \mathrm{t} \leq 0)
\end{aligned}
$$

Diamond:

$$
\begin{aligned}
f(x, y, z) & =\sin (x+y) \cos z+\cos (x-y) \sin z \\
& =\mathrm{t}(-1.5 \leq \mathrm{t} \leq 1.5)
\end{aligned}
$$

Double diamond:

$$
\begin{aligned}
f(x, y, z) & =\sin (x+y) \cos z+\cos (x-y) \sin z \\
& = \pm \mathrm{t}(-1.5 \leq \mathrm{t} \leq 0)
\end{aligned}
$$

where $x, y$ and $z$ are equal to $2 \pi X / \boldsymbol{a}, 2 \pi Y / \boldsymbol{a}$ and $2 \pi Z / \boldsymbol{a}$; $(X Y Z)$ are the coordinates of a specific point in the cubic unit cell; and $\boldsymbol{a}$ determines the cubic unit cell size. Taking gyroid as an example, a given $\mathbf{t}$ determines the morphology of a gyroid surface (composed of all points fulfilling the above formula) in the cubic network, which divides the cubic structure into two distinct interpenetrating regions. Here we define that $\mathbf{f}(x, y, z) \leqslant \mathbf{t}$ denotes filling with metal and that $\mathbf{f}(x$, $y, z)>\mathbf{t}$ denotes filling with air. Varying the value of $\mathbf{t}$ changes the composition of the materials, that is, the VF of the metal. ${ }^{28}$

\section{SERS detection}

The as-prepared GAPMMs were tailored into slices for Raman measurements, which were conducted under a Bruker Senterra R200-L Raman microscope operated with an Ar ion laser (532 nm) and $\mathrm{a} \times 50$ objective lens. The laser had a power of $2 \mathrm{~mW}$ and a beam diameter of $1 \mu \mathrm{m}$, and the integration time was $10 \mathrm{~s}$. The focal depth of the laser was $\sim 1 \mu \mathrm{m}$, which is relevant to the detection depth of the laser and smaller than the average thickness of the gyroid-structured samples measured from the TEM cross-section images $(2 \pm 0.5 \mu \mathrm{m})$. Therefore, the detection depth of the laser at each spot over the sample surface is approximately the same. The reproducibility was evaluated at thirty randomly chosen spots on the $3 \mathrm{~mm} \times 3 \mathrm{~mm}$ slice. The SERS mapping detection for analyzing the uniformity of GAPMMs- $4 \mathrm{~h}$ was performed by scanning an area of $20 \mu \mathrm{m} \times 20 \mu \mathrm{m}$ with a step of $1 \mu \mathrm{m}$.

\section{FDTD simulations}

For the FDTD simulations, the gyroid model was built using a combination of MATLAB 2010 and an FDTD program based on equation (1). The calculation server used for building the gyroid model and for the FDTD simulations was a Cluster Server with 480 Intel CPUs and 2T GB of internal storage. The unit cell size a was set to $360 \mathrm{~nm}$, to match the original template. The spatial resolution of the model was set to $\mathbf{a} / 2000$. For the mesh size, a convergence test was carefully performed and the mesh size was chosen to obtain a good trade-off between the required computer memory and the simulation time while ensuring convergence of the results. A plane-wave source with a wavelength of $532 \mathrm{~nm}$ (matching the laser source) was applied to illuminate perpendicular to the gyroid structure from the (100) direction. The boundary condition in the light incidence direction was set to provide a perfectly matched layer, and in the horizontal direction the boundary condition was set to the periodic boundary condition. Simulations in which the incident light showed both $s$ polarization and $p$ polarization were conducted. 


\section{RESULTS AND DISCUSSION}

Similar to the blue butterfly Morpho, C. rubi has drawn extensive attention due to its bright green color, originating from the gyroidstructured photonic crystals with a unit cell size of $\sim 360 \mathrm{~nm}$ and a VF of $17 \%(\mathbf{t}=1$, equation 1$){ }^{28-34}$ The gyroid-structured scales stack in a tiled manner on the ventral wings of C. rubi. Randomly oriented domains exist inside the scales, as shown in the optical image of a single gyroid scale (Figure 2a) and also demonstrated in the SEM and TEM images (Figures $2 \mathrm{~b}-\mathrm{d}$ ). 'Windows' framed by the longitudinal ridges and slender cross-ribs (Figures $2 \mathrm{e}$ and $\mathrm{f}$ ) homogeneously cover the external surface of the gyroid scales, which likely provide mechanical support for the gyroid components on the underside. Interestingly, the gyroid domains tend to be dense at the tip of the scale (zone A, Figure 2a) and gradually become sparse and even disappear at the root (zone B). ${ }^{35}$ Thus, a control experiment was also conducted in zone B, to eliminate the effects of the window structure.

SPR enhancement has been reported to dramatically increase with the reduction of the gap width, especially at a gap width of less than 5 $\mathrm{nm}{ }^{36,37}$ Although accurately evaluating the gap distribution in $3 \mathrm{D}$ gyroid networks with different VFs is difficult, simulated TEM projections along different plane directions can provide insight. Based on the TEM image of the cross-section of the biotemplate (Supplementary Figure S1), we selected several plane directions as examples and systematically investigated their gap distributions with an increasing VF via simulated TEM projections (Videos S1-S7). The $\mathbf{t}$ (from equation (1)) and VF values at which the number of nanogaps is maximized in the different plane directions are summarized in Supplementary Table S1. VF $\max$ differs for the different plane directions; however, these values are primarily located in the range of $70-85 \%$. In addition, $\mathrm{VF}_{\max }$ is subject to the actual unit cell size of the gyroid skeleton. Nevertheless, one point remains certain: proper control of the VF of GAPMMs can create a high-density 3Ddistributed nanogap matrix and hence considerably increase the SPR efficiency.

The TEM projection calculations not only exemplify the potential to acquire 3D-distributed nanogaps but also emphasize the importance of VF tuning during the templating process. To investigate the most suitable VF, a series of GAPMMs with different VFs were fabricated by controlling the $t_{\mathrm{d}}$ (see Supplementary Figure S3) in the electroless deposition process. The fabrication of GAPMMs based on a biotemplate involves three steps. The first two steps, amination of the biotemplate and $\mathrm{Au}$ nanoseed growth, were reported in our previous work. ${ }^{24}$ In the final electroless deposition process, a moderate reductant, diethanolamine, was used to guarantee the slow and homogeneous growth of Au films. A proper concentration of halide ions $\left(\mathrm{Cl}^{-}\right)$was also included to control the morphology of the Au films. ${ }^{38}$ For a closer view of the GAPMMs under the window structure, SEM and TEM images of the cross-sections of the scales with different $t_{\mathrm{d}}$ are shown in Figures $3 \mathrm{a}-\mathrm{f}$. At $t_{\mathrm{d}}=1 \mathrm{~h}$, Au films with a thickness of $\sim 17 \mathrm{~nm}$ begin to form and homogeneously cover the gyroid skeleton. Upon increasing the $t_{\mathrm{d}}$ to $5 \mathrm{~h}$, the $\mathrm{Au}$ films grow uniformly. Notably, during the uniform growth of the Au films, the gyroid morphology and the interconnectivity of the channels were maintained, as confirmed by comparing the TEM images of the crosssections (Figure 3, A column) with the simulated TEM projections (Figure 3, B column) of the corresponding VFs. The interconnectivity of the helices with different $t_{d}$ is also indicated by the SEM images of the cross-sections (Figure 3, C column) and the simulated gyroid networks with the corresponding VFs (Figure 3, D column). The widths between the interconnected channels slowly and homogeneously decreased with Au deposition, until all the channels were fully blocked after a $t_{d}$ of $6 \mathrm{~h}$. A fitted growth curve of the Au films is depicted in Supplementary Figure S4. The thickness of the Au films demonstrates a strong linear relationship with $t_{d}(1-5 \mathrm{~h})$. In fact, when the VF is higher than $98 \%$ ( $\mathbf{t}>1.413)$, a so-called pinch-off surface is formed, which breaks the channel interconnectivity. ${ }^{39}$ The residual channels are then separated into isolated pores (as shown in Supplementary Figure S2) and shielded from the electroless deposition bath. Therefore, we can only approach but never reach a VF of $100 \%$ by extending the $t_{\mathrm{d}}$. Detailed structural parameters of these GAPMMs are listed in Table 1. The extinction spectra of these GAPMMs were also measured using a microscope spectrometer (Supplementary Figure S7). A broad absorption band is found in the wavelength range of $450-500 \mathrm{~nm}$, and the absorption between $500 \mathrm{~nm}$ and $700 \mathrm{~nm}$ decreases with increasing $t_{\mathrm{d}}$, which is in good agreement with previously reported results. ${ }^{23}$ The absence of characteristic absorption peaks corresponding to the gyroid structure and the formation of nanogaps may originate from the scattering of the window structure and structural defects, as well as the existence of nanogaps with different widths.

To quantitatively analyze the nanogap distributions in the GAPMMs with different $t_{\mathrm{d}}$, we manually measured the nanogap widths from the TEM images, which are summarized in Supplementary Table S2. Some of the nanogaps (shown in Supplementary Figure S5) are also directly labeled on the TEM cross-section images. The gap widths between the interconnected channels continuously decreased as the $t_{\mathrm{d}}$ increased from 1 to $3 \mathrm{~h}$. Then, the number of nanogaps with widths ranging from 3 to $25 \mathrm{~nm}$ reached a maximum at a $t_{d}$ of $4 \mathrm{~h}$. Afterwards, excess deposition of Au largely decreased the number of nanogaps at a $t_{\mathrm{d}}$ of $5 \mathrm{~h}$, until finally all the channels were blocked at a $t_{\mathrm{d}}$ of $6 \mathrm{~h}$. Therefore, the SPR enhancement of GAPMMs- $4 \mathrm{~h}$ is anticipated to be higher than that of the GAPMMs with other $t_{d}$ values. Although determination of the distribution of nanogaps in all three dimensions is difficult, the nanogap distribution from the TEM cross-section can provide insight into that of the two other dimensions. Despite the existence of nanogaps with different widths, the nanogaps are periodically distributed among the interconnected helices with their extension in 3D space. The SEM image acquired from the underside of the gyroid-structured scale provides direct understanding of the 3D-interconnected helices in GAPMMs-4 h (as shown in Supplementary Figure S6a).

To verify this hypothesis, the SERS detection of CV molecules using the GAPMM substrates with different $t_{\mathrm{d}}$ was performed to monitor their SPR enhancements. Au butterfly wings were tailored into $3 \mathrm{~mm} \times 3 \mathrm{~mm}$ slices and directly used as substrates for SERS detection without removing the original chitin skeletons, as it has been reported that the original skeleton does not have a substantial influence on SERS detection at certain incident laser wavelengths, that is, $514 \mathrm{~nm}$ and $532 \mathrm{~nm} .{ }^{8,40}$ A CV alcohol solution $(25 \mu \mathrm{l})$ with a concentration of $10^{-4} \mathrm{M}$ was carefully dropped onto the $3 \mathrm{~mm} \times 3 \mathrm{~mm}$ slices and allowed to dry naturally at room temperature. A $532 \mathrm{~nm}$ incident laser was then applied to conduct SERS detection. The SERS signals detected on the GAPMM substrates in zone A with different $t_{\mathrm{d}}$ are shown in Figure 4a. The SERS enhancement displayed slow growth as the $t_{\mathrm{d}}$ increased from 1 to $3 \mathrm{~h}$, corresponding to a slow decrease in the channel width with increasing VF. The enhancement then reached a maximum with a sharp increase as the $t_{d}$ reached $4 \mathrm{~h}$. Subsequently, the SERS enhancement began to decline at a $t_{\mathrm{d}}$ of $5 \mathrm{~h}$, as the deposition of excess Au reduced the number of nanogaps. Finally, the enhancement experienced a considerable decrease as entire channels were blocked at a $t_{\mathrm{d}}$ of $6 \mathrm{~h}$. 


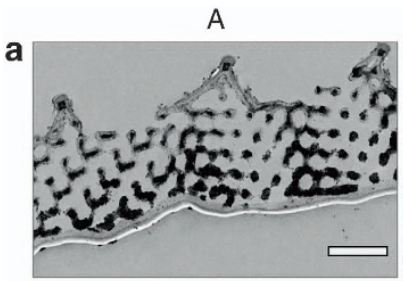

B
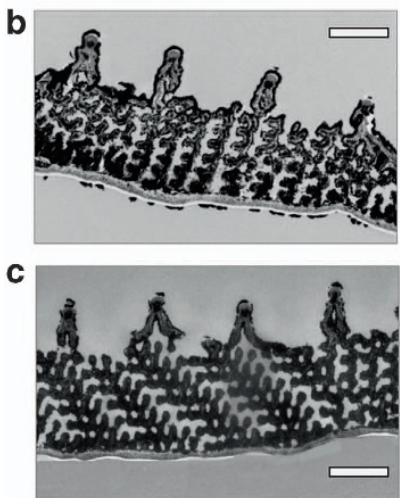

d
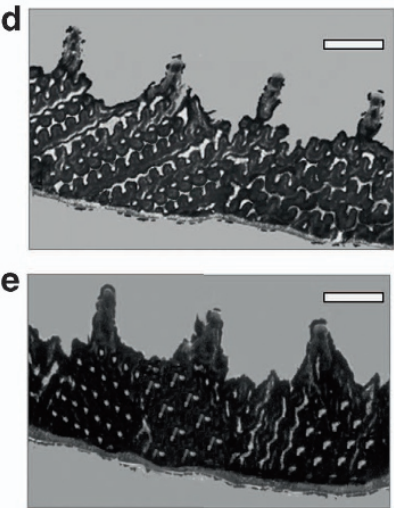

f

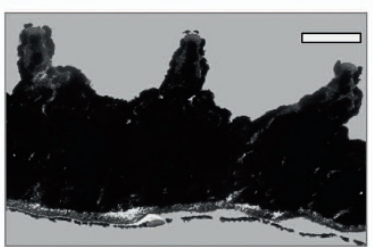

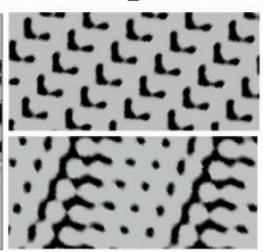
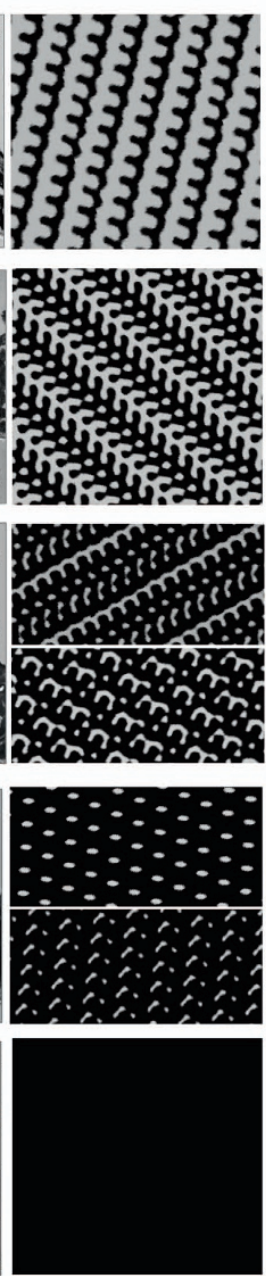

C
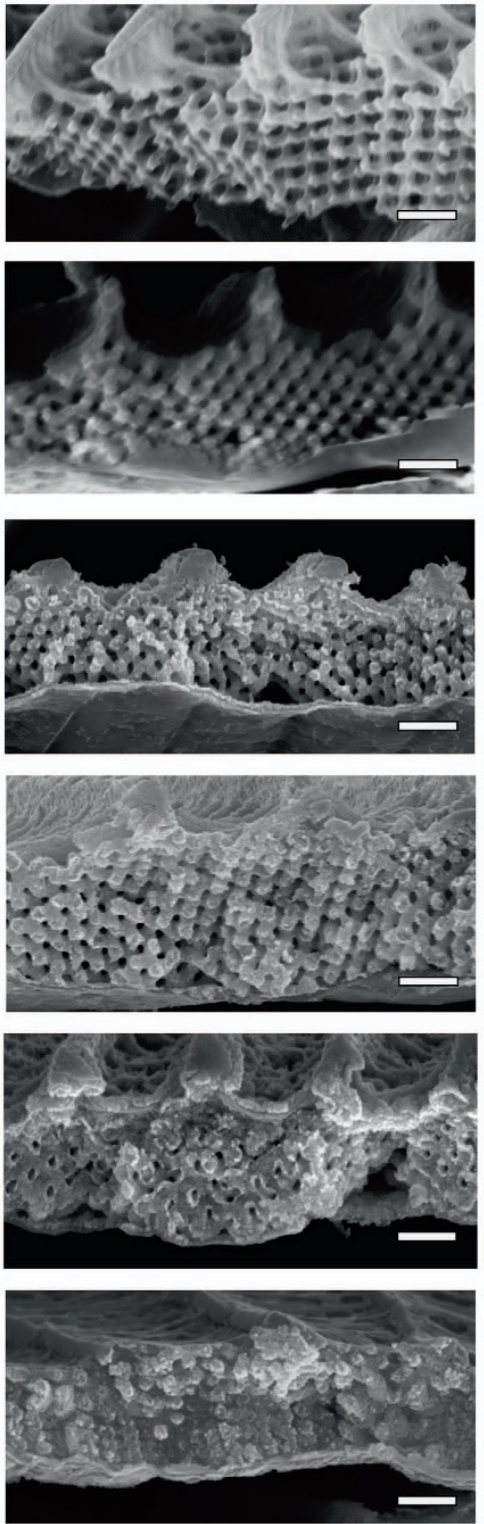

$\mathrm{D}$
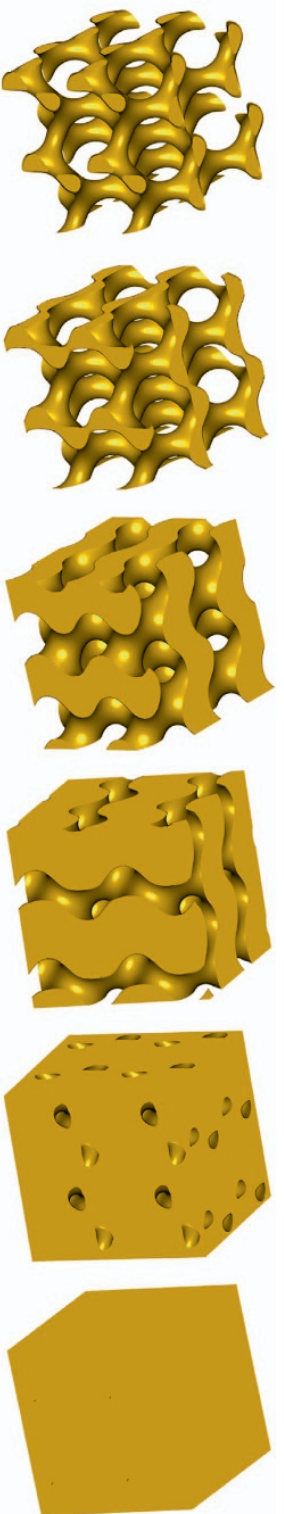

Figure 3 Experimental and simulated images of the cross-sections of zone A with different $t_{\mathrm{d}}$. TEM images (A column), TEM projections (B column), SEM

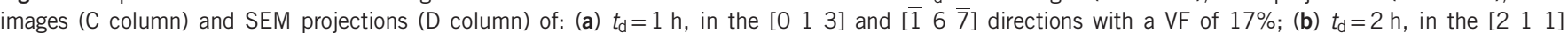
direction with a VF of $34 \%$; (c) $t_{d}=3 \mathrm{~h}$, in the [0 3 5] direction with a VF of $53 \%$; (d) $t_{d}=4 \mathrm{~h}$, in the [3 25 ] and [2 4 9] directions with a VF of 79\%; (e)

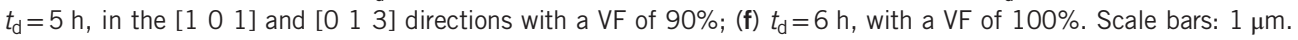

Table 1 Structural parameters of GAPMMs with different $t_{\mathrm{d}}$

\begin{tabular}{lcccccc}
\hline DT $(\mathrm{h})$ & 1 & 2 & 3 & 4 & 5 & 6 \\
Thickness (nm) & 17 & 32 & 61 & 75 & 89 & 1 \\
VF $(\%)^{a}$ & 23 & 34 & 53 & 79 & 91 & 100 \\
$t^{b}$ & 0.85 & 0.5 & -0.1 & -0.9 & -1.2 & -1.5 \\
\hline
\end{tabular}

Abbreviations: DT, deposition time; GAPMM, gyroid-structured Au periodic metallic material; TEM, transmission electron microscopy; $V F$, volume fraction.

aThe VFs of the GAPMMs with different $t_{\mathrm{d}}$ values in the table are the averages of several different plane directions in the TEM images of the cross-section.

bThe $t$ values were evaluated using equation (1) based on the corresponding VFs.

To further investigate the SPR enhancement of the GAPMMs near a $t_{\mathrm{d}}$ of $4 \mathrm{~h}$, GAPMMs with different $\mathrm{t}_{\mathrm{d}}$ between 3 and $5 \mathrm{~h}$ (in increments of $20 \mathrm{~min}$ ) were fabricated and their SERS performances were studied in the same manner. As shown in Figure $4 \mathrm{~b}$, the SPR enhancement quickly and almost linearly increased when the $t_{\mathrm{d}}$ increased from 3 to $4 \mathrm{~h}$, before a maximum was acquired at a $\mathrm{t}_{\mathrm{d}}$ of $4 \mathrm{~h}$. Afterwards, the enhancement began to decline as the $t_{d}$ increased to $5 \mathrm{~h}$. Control experiments were also conducted in zone B (Figure 4c). In contrast to the trend in zone A, the SERS enhancement experienced stable growth as the $t_{d}$ increased from 1 to $6 \mathrm{~h}$ (Figure $4 \mathrm{~d}$ ), but the enhancement was obviously smaller than that in zone A. In particular, at a $t_{\mathrm{d}}$ of $4 \mathrm{~h}$, the enhancement in zone A was 12 times greater than that in zone B, which distinctly excludes the impact of the window structure, exhibiting the superiority of the gyroid structure.

Furthermore, we conducted FDTD simulations to gain insight into the SPR mechanism of the GAPMMs with different VFs. The unit cell size used for the simulation was set to $360 \mathrm{~nm}$ based on the original 

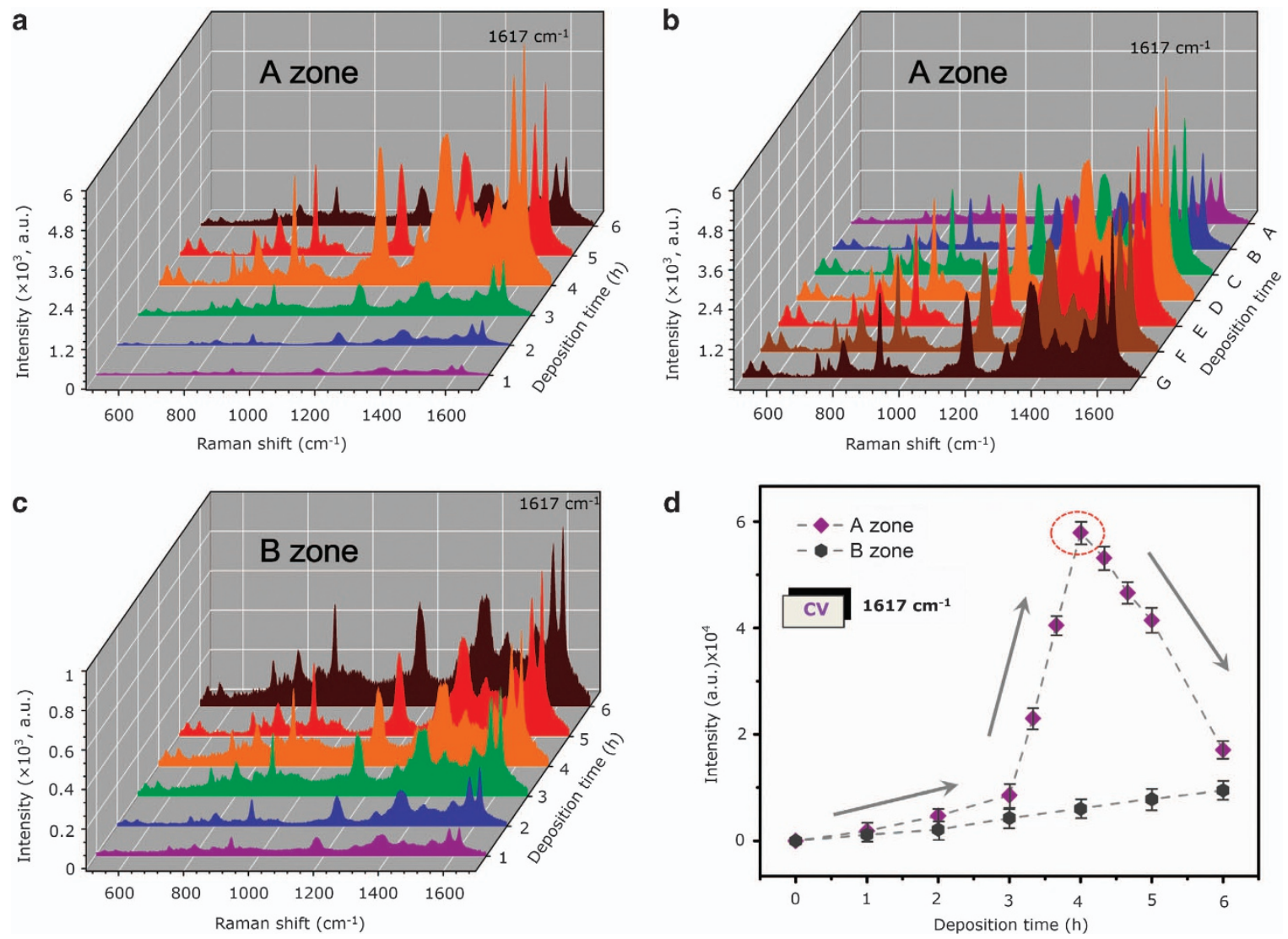

Figure 4 (a) SERS signals of $10^{-4} \mathrm{M}$ CV detected from the GAPMMs in zone A with $t_{\mathrm{d}}$ of $1,2,3,4,5$ and $6 \mathrm{~h}$. (b) SERS signals of $10^{-4} \mathrm{M}$ CV detected from the GAPMMs in zone A with $t_{d}$ between 3 and $5 \mathrm{~h}$ : A $3 \mathrm{~h} ; \mathrm{B} 3 \mathrm{~h}$ and $20 \mathrm{~min}, \mathrm{C} 3 \mathrm{~h}$ and $40 \mathrm{~min} ; \mathrm{D} 4 \mathrm{~h} ; \mathrm{E} 4 \mathrm{~h}$ and $20 \mathrm{~min} ; \mathrm{F} 4 \mathrm{~h}$ and $40 \mathrm{~min} ; \mathrm{G} 5 \mathrm{~h}$. (c) SERS signals of $10^{-4} \mathrm{M}$ CV detected from zone B of the gyroid-structured scales with different $t_{\mathrm{d}}$. (d) Variation trends of the peak CV SERS signals at wavenumber $1617 \mathrm{~cm}^{-1}$ versus $t_{d}$ detected from both zone $A$ and zone $B$.
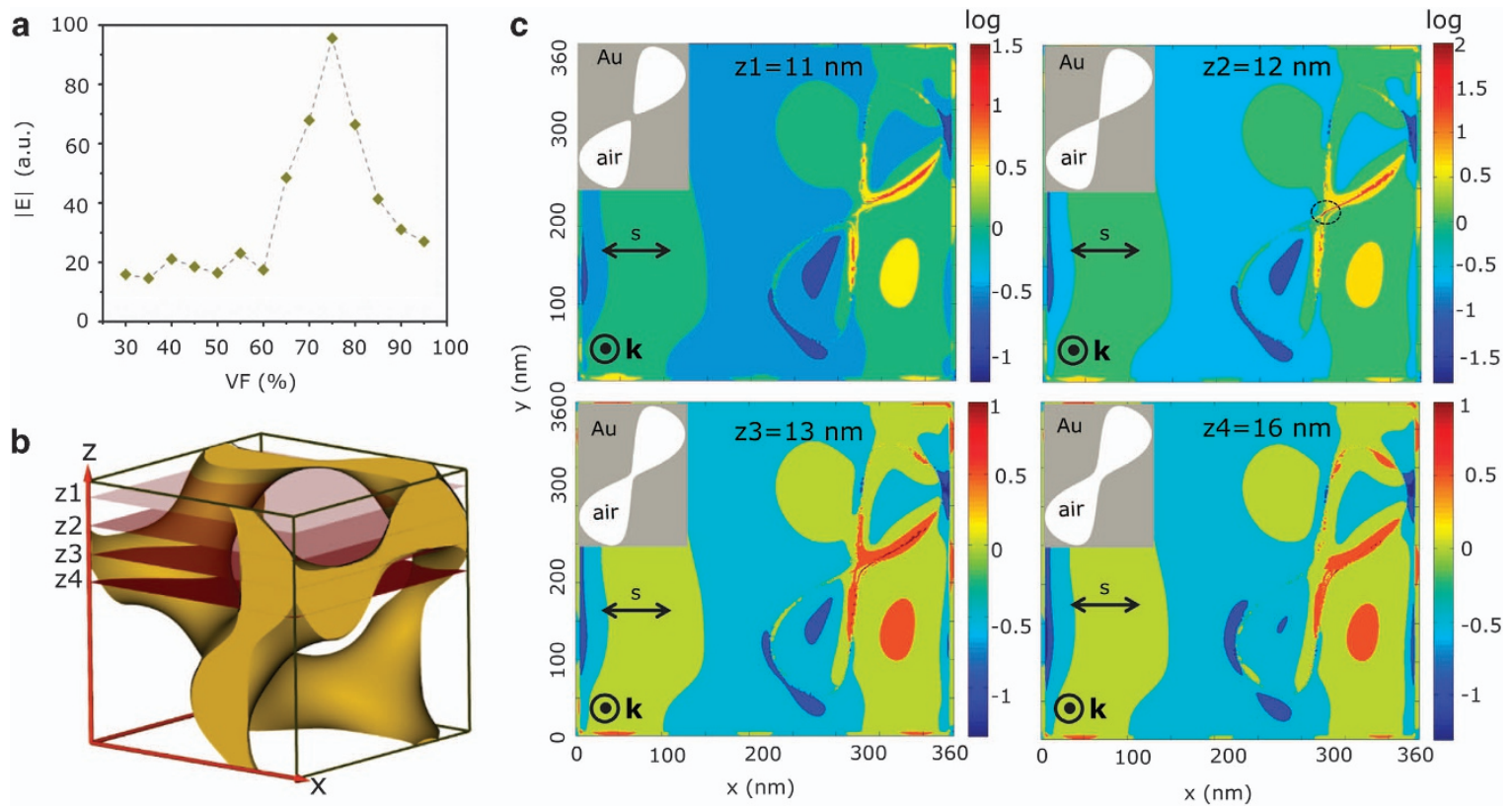

Figure 5 (a) Calculated electric field enhancement IEI using FDTD. The intensity of the incident electric field is 1. (b) Relative positions of the four energy maps labeled on the gyroid model with one unit cell and a VF of 34\%. (c) Exponential distributions of the calculated electric field enhancements on the [100] plane directions with different $z$ depth in at a VF of $75 \%$ based on the gyroid model. The inset shows the TEM projections with corresponding $z$ depths in the [100] direction. 
a

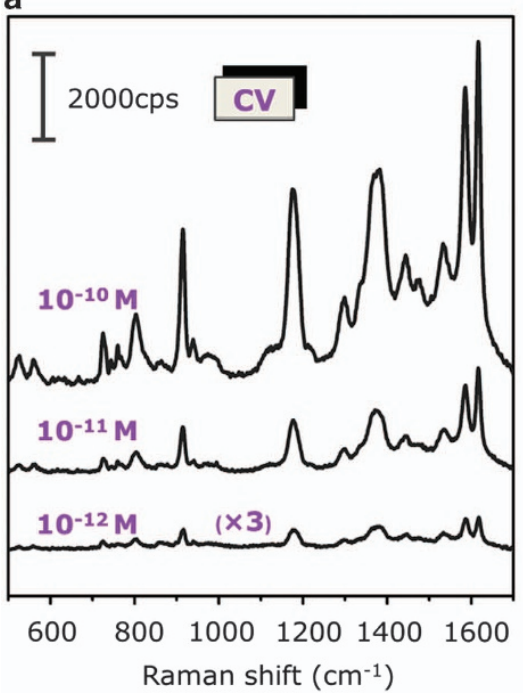

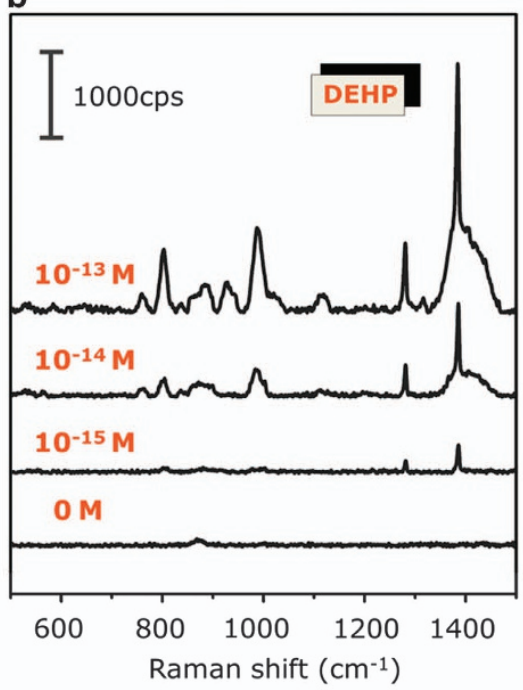

Figure 6 Raman detection of $\mathrm{CV}$ and DEHP based on GAPMMs-4 h. (a) SERS signals of $\mathrm{CV}$ with concentrations of $10^{-10}$, $10^{-11}$ and $10^{-12} \mathrm{M}$ (top to bottom). (b) SERS signals of DEHP with concentrations of $10^{-13}, 10^{-14}, 10^{-15}$ and $0 \mathrm{~m}$ (top to bottom).

template. As shown in Figure 5a, the simulated variation in the SPR enhancement versus VF was in good agreement with the experimental results of the GAPMMs. The electric field intensity did not greatly change as the VF increased from $25 \%$ to $60 \%$. At a VF of $60 \%$, the SPR enhancement began to quickly increase before reaching a maximum at a VF of $75 \%$, which is very close to the experimental value of $79 \%$. Upon further increasing the VF, the SPR enhancement started to decrease until reaching a VF of 95\%. The energy map inside one gyroid cubic unit cell with a VF of $75 \%$ was also calculated using FDTD. The SPR enhancement reached as high as $10^{8}$ using incidence light with both $\mathrm{p}$ and $\mathrm{s}$ polarization. For $s$ polarized light, the exponential distribution of the electric field on the four twodimensional planes in the [100] direction with different $\mathrm{z}$-axis depths is shown in Figure $5 c$ (the simulation results for $\mathrm{p}$ polarization are shown in Supplementary Figure S13 for reference). The insets of these figures show the cross-section projections at corresponding $z$-axis depths. The gray region represents gold and the blank area represents air. Among these projections, the plane on $\mathrm{z} 2$ has a position with a narrow nanogap, while $\mathrm{z} 1, \mathrm{z} 3$ and $\mathrm{z} 4$ are planes near $\mathrm{z} 2$ (the relative position of these planes is shown in Figure 5b). From these energy maps, we find that the SPR enhancement is much higher in the plane at depth $\mathrm{z} 2$ with a narrow gap than at the other planes at depths $\mathrm{z} 1, \mathrm{z} 3$ and $\mathrm{z} 4$. Therefore, we can conclude that the highest electric field enhancement was closely located around the nanogaps and that gaps with larger widths contribute much less to the enhancement.

Moreover, in addition to the highest SPR enhancement at the position with a narrow gap, a considerable SPR enhancement is also found around the internal interface between $\mathrm{Au}$ and air near the nanogap, as shown in Figure $5 \mathrm{c}$, which was deduced to originate from the interconnected helices. First, the interconnected helices largely increase the spatial utilization compared with a linear structure. By properly tuning the VF, we can acquire high-density hotspots in all three dimensions. Second, we proved in our previous work that a $3 \mathrm{D}$ hierarchical configuration is important to increasing the scattering cross-section, which enhances the multi-scattering of incident light and increases light absorption, finally contributing to a high SPR enhancement. ${ }^{41}$ Therefore, the 3D bicontinuous interconnected helices will increase the scattering cross-section of incidence light, which further increases the SPR enhancement. Although reducing the channel width by continuously depositing Au will surely lead to a decrease in the scattering cross-section, a competition exists between the nanogap density and the scattering cross-section upon increasing the VF. Theoretically, we can determine a suitable VF to balance the two factors and acquire a much higher SPR enhancement.

Subsequently, the SERS sensitivity, reproducibility and uniformity of GAPMMs- $4 \mathrm{~h}$ were systematically evaluated. We first used the GAPMM substrates to investigate the detection limit of CV molecules. As shown in Figure 6a, the SERS spectrum is still distinctly obtained at a concentration down to $10^{-12} \mathrm{M}$ with the use of $25 \mu \mathrm{l}$ of solution, which is four orders of magnitude lower than that of gyroid-structured plasmonic materials templated from block polymer systems ${ }^{15}$ and two orders of magnitude lower than that of the commercial SERS substrate Q-SERS (as shown in Supplementary Figure S11); however, the cost of GAPMMs-4 $\mathrm{h}$ is far less than Q-SERS. According to the assumptions and procedures in published works, ${ }^{14}$ the average enhancement factor for GAPMMs- $4 \mathrm{~h}$ is estimated to be $0.8 \times 10^{9}$ under $532 \mathrm{~nm}$ laser excitation (as shown in the supporting information). It is noteworthy that the evaluated enhancement factor based on experimental results is approximately the same order of magnitude as that obtained from the FDTD simulations $\left(\sim 10^{8}\right.$, proportional to the fourth power of the electric field intensity, $\left.|\mathrm{E}|^{4}\right)$. Considering the chemical enhancement ${ }^{42}$ and possible contribution from the rough surface of real Au films, the simulated result is in good agreement with the experimental result.

Afterwards, to examine the capacity of GAPMMs- $4 \mathrm{~h}$ to monitor food and environmental safety, we chose DEHP with an ultralow concentration for detection, as DEHP is the most frequently used plasticizer in perfumes, children toys, paints, household itemsand so on, and poses a high risk when in drinking water. The Raman spectra (532 nm incident laser) of DEHP in concentrations ranging from $10^{-13}$ to $10^{-15} \mathrm{M}$ are shown in Figure $6 \mathrm{~b}$. The characteristic Raman peaks of DEHP can still be distinguished at a concentration down to $10^{-15}$ M. Compared with other novel substrates with sensitivities down to $\mathrm{fM}$, GAPMMs are not only facile and inexpensive to fabricate but also freestanding, mechanically robust and easy to transfer between various application environments. ${ }^{7}$ Therefore, the super-sensitive, freestanding and cost-effective GAPMM substrates hold great potential in ultralow concentration detection for drug security, food safety, environmental protection and so on. 
a

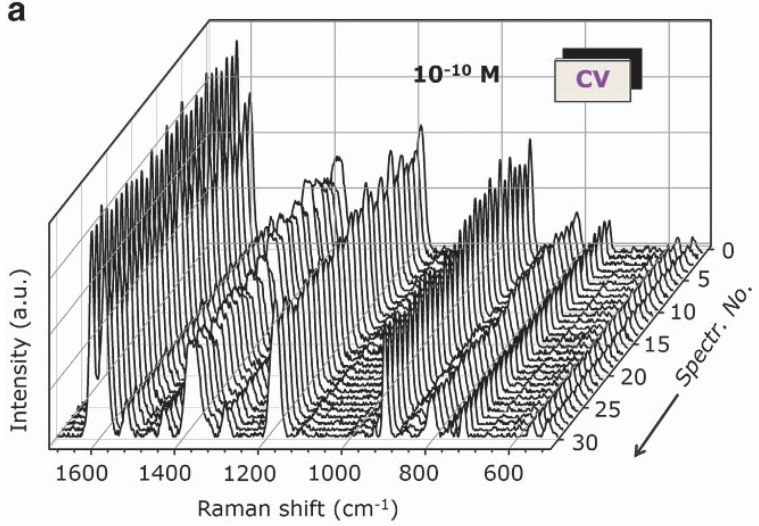

C

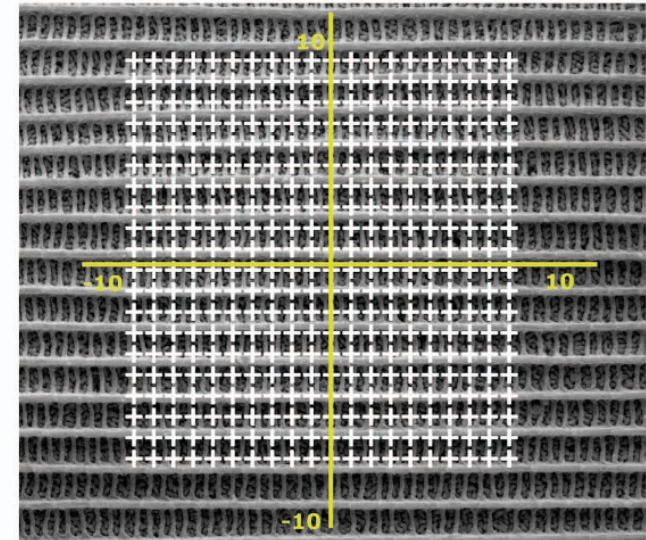

b

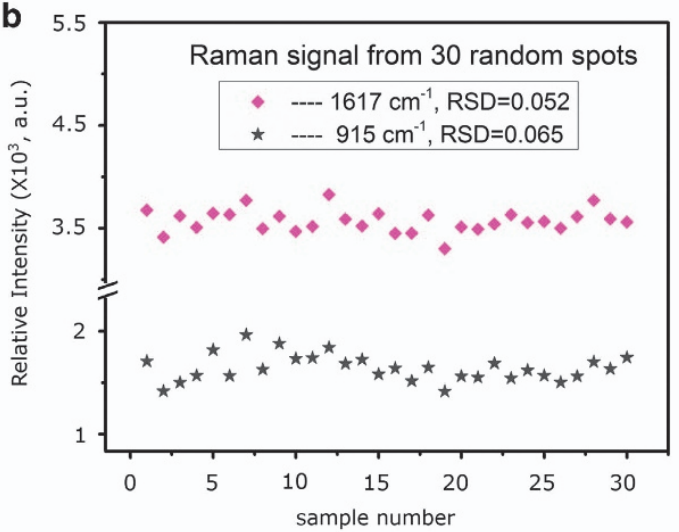

d

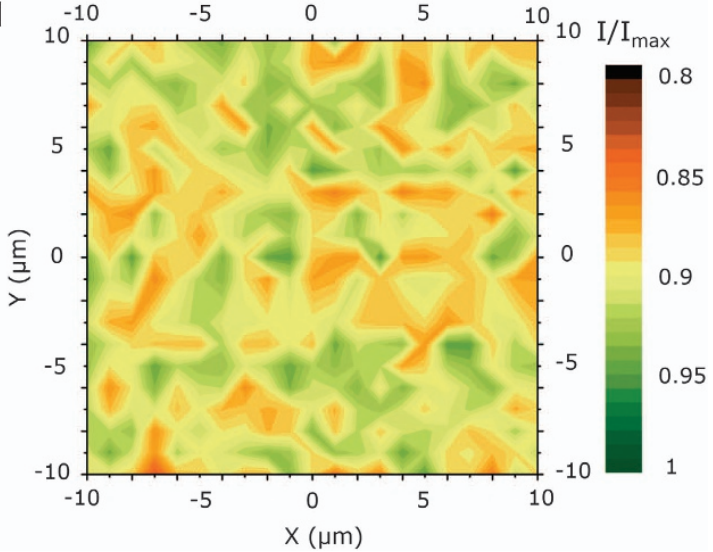

Figure 7 Reproducibility and uniformity analysis of GAPMMs-4 h based on CV molecules. (a) Raman signals (10-10 M) obtained from 30 different gyroid scales in A zone. It is noteworthy that the concentration used for the reproducibility evaluation is one order lower than that of our previous work. (b) Raman intensity distribution of the 30 spectra at the peak wavenumbers 915 and $1617 \mathrm{~cm}^{-1}$, with relative s.d. (RSDs) of 0.065 and 0.052 , respectively. (c) Topview SEM image of GAPMMs-4 $\mathrm{h}$ with the illustration of SERS mapping experiment measured by scanning a $20 \mu \mathrm{m} \times 20 \mu \mathrm{m}$ area with a laser spot diameter of $1 \mu \mathrm{m}$ at a step-size of $1 \mu \mathrm{m}$. (d) Normalized SERS intensity distribution of the Raman signal (CV, 10-10 $\mathrm{M}$ ) at the peak wavenumber $1617 \mathrm{~cm}^{-1}$ acquired from the mapping.

Furthermore, the reproducibility of the SERS enhancement by this platform was investigated by randomly selecting 30 spots on 30 different gyroid-structured scales in zone A for the detection of CV $\left(10^{-10} \mathrm{M}\right)$. As shown in Figures $7 \mathrm{a}$ and $\mathrm{b}$, the 30 SERS spectra demonstrated superior consistency with a relative s.d. of $5.2 \%$ and $6.5 \%$ at a peak wavenumber of 1617 and $915 \mathrm{~cm}^{-1}$, respectively. These results are comparable to those of many other SPR substrates, whereas the detection concentration is one or two orders of magnitude lower. $^{24,43}$ Meanwhile, to investigate the influence of the randomly oriented distribution of domains on the uniformity of SPR enhancement by the GAPMMs, a point-by-point SERS mapping measurement was conducted on a $20 \mu \mathrm{m} \times 20 \mu \mathrm{m}$ area in zone A for the detection of $\mathrm{CV}\left(10^{-10} \mathrm{M}\right)$. The step lengths in both the $X$ and $Y$ directions were $1 \mu \mathrm{m}$. As shown in Figures $7 \mathrm{c}$ and $\mathrm{d}$, the Raman intensities acquired on all these $20 \times 20$ spots at a peak wavenumber of $1649 \mathrm{~cm}^{-1}$ demonstrate high uniformity. We can conclude that the anisotropy of these domains does not have a substantial influence on the SPR efficiency of the gyroid structure. As randomly oriented gyroid domains exist in both natural templates and self-assembled motifs in block polymer systems, confirmation of the uniform SPR enhancement over anisotropic domains is of great significance for potential plasmonic applications.

In addition, the reusability of the GAPMMs- $4 \mathrm{~h}$ substrate was evaluated by repeatedly depositing and removing CV molecules from the substrate. As shown in Figures $8 \mathrm{a}$ and b, after 15 cycles, the intensity of the Raman signal at a peak wavenumber of $1617 \mathrm{~cm}^{-1}$ maintains $95 \%$ of the intensity of the first measurement, which means that the gyroid-structured plasmonic substrates are quite stable and robust. In addition, as discussed in previously, the most suitable VF to acquire hotspots with high density depends on the unit cell size of the gyroid structure. As the unit cell sizes of different gyroid domains in different butterflies may slightly differ from one another, we fabricated GAPMM- $4 \mathrm{~h}$ substrates based on 10 different $C$. rubi butterflies and then detected the Raman signal of $10^{-10} \mathrm{M} C V$ using these substrates to investigate the difference between butterflies. As shown in Figures $8 \mathrm{c}$ and $\mathrm{d}$, slight fluctuation within the expected limit is observed among these spectra. The relative s.d. calculated from the intensities at peak wavenumbers of 1617 and $915 \mathrm{~cm}^{-1}$ are $7.1 \%$ and $5.8 \%$, respectively. These results are comparable with those of many other hierarchical plasmonic substrates., $9,24,43$

More importantly, the mechanism presented here can act as a guideline for the future design and selection of SPR substrates. The existence of several other bicontinuous interconnected structures has been confirmed in various biological systems and self-assembly systems, such as the diamond structure found in the brilliantly green weevil Lamprocyphus augustus ${ }^{25}$ and the double-gyroid, ${ }^{44}$ stripedgyroid $^{26}$ and double-diamond ${ }^{45}$ structures in block polymer systems. Figure 8 depicts the nanogap distribution in the two-dimensional 

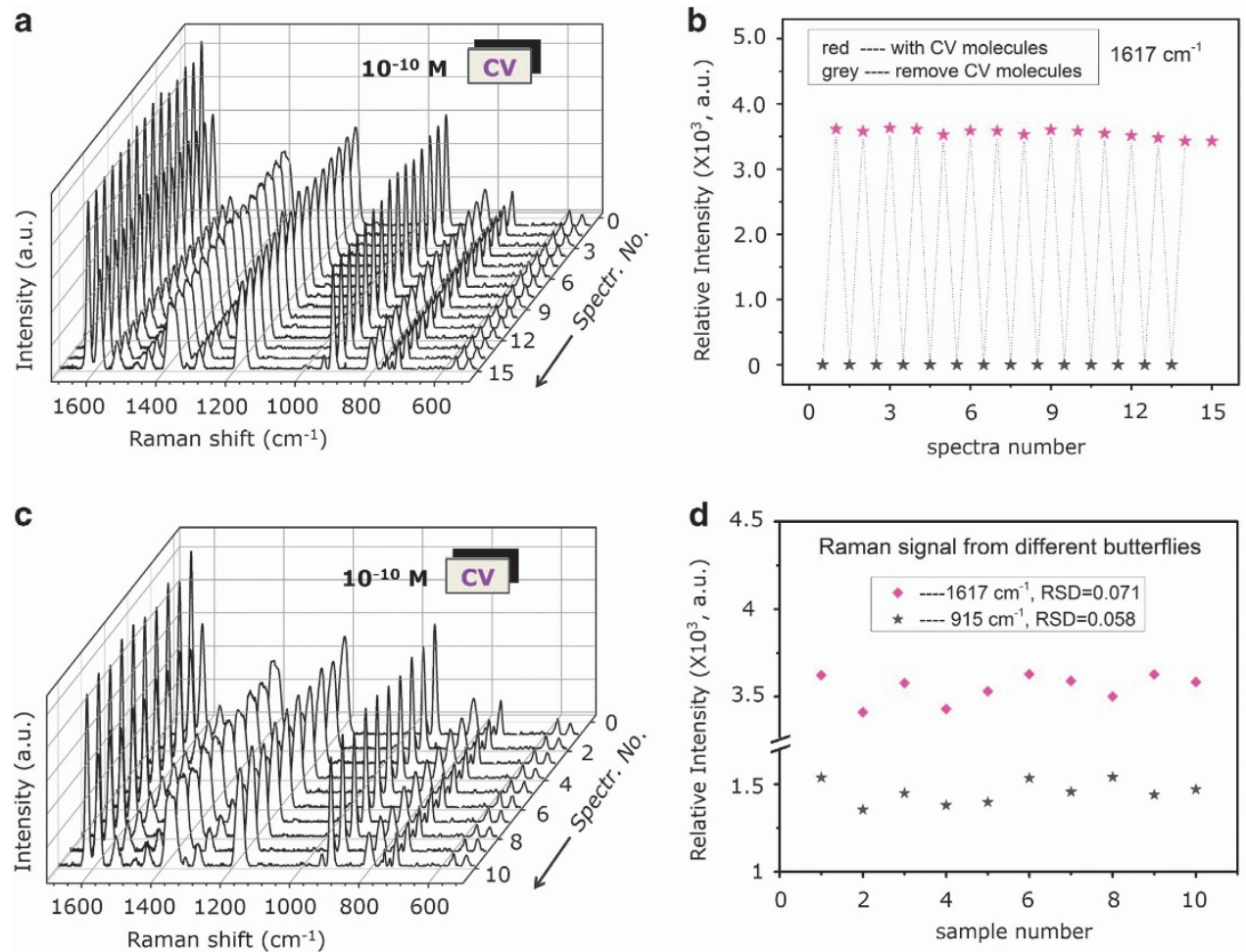

Figure 8 (a) Fifteen CV $\left(10^{-10} \mathrm{M}\right)$ SERS spectra for reusability analysis of the GAPMMs-4 h substrate. (b) Raman intensity distribution of the 15 spectra in a at the peak wavenumber $1617 \mathrm{~cm}^{-1}$. (c) Ten CV $\left(10^{-10} \mathrm{M}\right)$ SERS spectra obtained from the GAPMMs-4 h substrates templated from 10 different butterflies. (d) Raman intensity distribution of the 10 spectra in $\mathbf{c}$ at the peak wavenumbers 915 and $1617 \mathrm{~cm}^{-1}$, with relative s.d. (RSDs) of 0.058 and 0.071 , respectively.

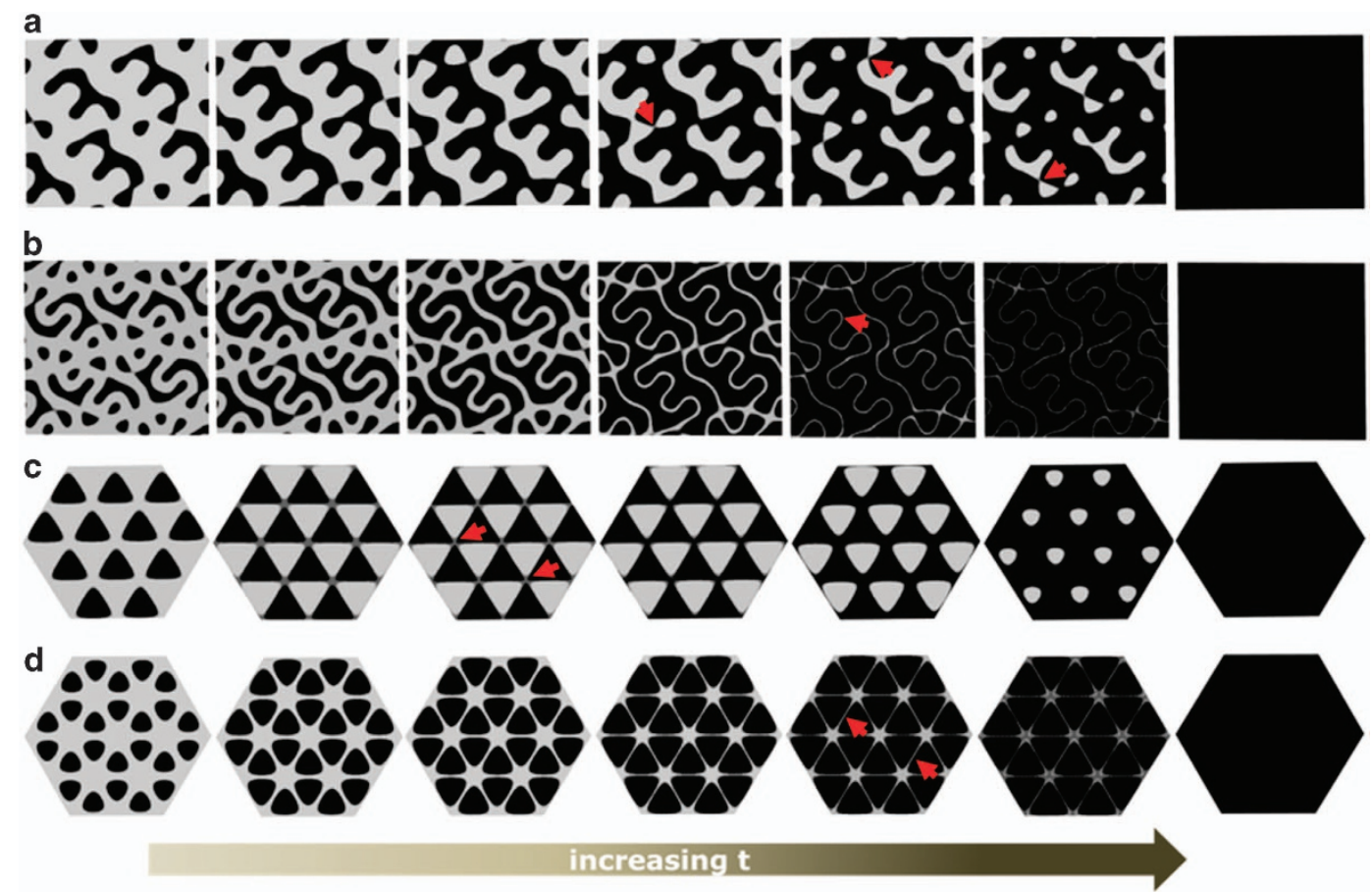

Figure 9 Simulated TEM projections of different bicontinuous structures: (a) gyroid and (b) double-gyroid structures along the [2 4 9] direction; (c) diamond and (d) double-diamond structures along the $\left[\begin{array}{lll}1 & 1 & 1\end{array}\right]$ direction, with an increasing VF. For the gyroid and diamond structures, note that the value of $\mathbf{t}$ varies in the range between -1.5 and 1.5 , and that the nanogaps originate from the approaching of one network by increasing its VF. However, for the double gyroid and double diamond structures, $\mathbf{t}$ varies in the range between -1.5 and 0 , and the nanogaps originate from the approaching of the two centrosymmetric networks with the increasing of their VFs. 
cross-sections of the diamond structure versus VFs. The trend is similar to that of the gyroid structure. In particular, the double-gyroid and double-diamond structures, which are composed of two sets of centrosymmetric gyroid and diamond networks, respectively, possess even higher spatial utilization. Thus, an even higher nanogap density can be acquired with proper control of the VF, as indicated by the red arrows in Figure 9 and Supplementary Figure S15.

\section{CONCLUSION}

In summary, we proved the existence of a high-density 3D-distributed nanogap matrix among the interconnected channels in GAPMMs- $4 \mathrm{~h}$ by both experimental and theoretical analysis. GAPMMs- $4 \mathrm{~h}$ not only possessed ultra-high SPR sensitivity but also demonstrated superior reproducibility, uniformity and stability. The SERS detection limit for DEHP, the most frequently used plasticizer, which poses high risks in food and environmental safety, was as low as $1 \mathrm{fM}$. Compared with other PMM substrates fabricated by either top-down or templating methods, gyroid-structured plasmonic materials possess at least three advantages. First, the bicontinuous interconnected helices fully utilize all three spatial dimensions, enabling us to engineer hotspots with high density in three dimensions. Second, the 3D interconnected helices largely increase the scattering cross-section, which facilitates the absorption of incident light and further increases the SPR efficiency. Consequently, the SPR enhancement of the GAPMMs is much higher than that of SPR substrates with hotspots distributed in two dimensions, such as the nanoridge structure in $x-z$ dimensions ${ }^{22}$ and the lithographically patterned nanoparticle arrays in $x-y$ dimensions. ${ }^{23}$ Last but not least, the interconnected channels in the gyroid structure have been proved to be a 'highway' for efficient mass and electron transport by both experiments and simulations. ${ }^{46,47}$ Functional materials with gyroid structures have been demonstrated to perform well in solar cells, ${ }^{48}$ optoelectronic devices, ${ }^{49}$ catalysis $^{50}$ and cell culturing. ${ }^{51}$ Therefore, in addition to functioning as plasmonic sensors, these materials also hold potential for applications requiring efficient matter transport, as well as superior SPR performance, such as photocatalysis, plasmonic solar cells and heat-induced evaporation. A very recent work demonstrated the possibility of predicting the unit cell size of the gyroid structure in self-assembly systems. ${ }^{52}$ Combined with theoretical TEM projection analysis, the most suitable VF for superior SPR performance can be determined, which can in turn guide the engineering of 3D-distributed nanogaps in bicontinuous systems with versatile unit cell sizes using the electroless deposition method developed in this work.

\section{CONFLICT OF INTEREST}

The authors declare no conflict of interest.

\section{ACKNOWLEDGEMENTS}

This work was supported by the National Natural Science Foundation of China (number 51572169), the Shanghai Rising-Star Program (16QA1402400), the Shanghai Science and Technology Committee (15ZR1422400, 14JC1403300 and 14520710100), the National Basic Research Program of China (973 Program, number 2011CB922200), the National Key Research and Development Program (number 2016YFB0701200), the National Natural Science Foundation of China (grant number 51602205) and the Science and Technology Innovation Commission of Shenzhen (grant number JCYJ20160520165724531).

\section{PUBLISHER'S NOTE}

Springer Nature remains neutral with regard to jurisdictional claims in published maps and institutional affiliations
1 Reineck, P., Lee, G. P., Brick, D., Karg, M., Mulvaney, P. \& Bach, U. A solid-state plasmonic solar cell via metal nanoparticle self-assembly. Adv Mater 24, 4750-4755 (2012).

2 Tian, C., Ding, C., Liu, S., Yang, S., Song, X., Ding, B. \& Fang, J. Nanoparticle attachment on silver corrugated-wire nanoantenna for large increases of surfaceenhanced Raman scattering. ACS Nano 5, 9442 (2011).

3 Fang, J., Du, S., Lebedkin, S., Li, Z., Kruk, R., Kappes, M. \& Hahn, H. Gold mesostructures with tailored surface topography and their self-assembly arrays for surface-enhanced Raman spectroscopy. Nano Lett 10, 5006-5013 (2010).

$4 \mathrm{Ma}, \mathrm{C}$. , Gao, Q., Hong, W., Fan, J. \& Fang, J. Real-time probing nanopore-in-nanogap plasmonic coupling effect on silver supercrystals with surface-enhanced Raman spectroscopy. Adv Funct Mater 27, 1603233 (2017).

5 Liu, H., Yang, Z., Meng, L., Sun, Y., Wang, J., Yang, L., Liu, J. \& Tian, Z. Three-dimensional and time-ordered surface-enhanced Raman scattering hotspot matrix. J Am Chem Soc 136, 5332-5341 (2014).

6 Ou, F. S., Hu, M., Naumov, I., Kim, A., Wu, W., Bratkovsky, A. M., Li, X., Williams, R. S. \& Li, Z. Hot-spot engineering in polygonal nanofinger assemblies for surface enhanced Raman spectroscopy. Nano Lett 11, 2538-2542 (2011).

7 Peng, B., Li, G., Li, D., Dodson, S., Zhang, Q., Zhang, J., Lee, Y. H., Demir, H. V., Ling, X. Y. \& Xiong, Q. Vertically aligned gold nanorod monolayer on arbitrary substrates: self-assembly and femtomolar detection of food contaminants. ACS Nano 7, 5993-6000 (2013)

8 Xu, B-B, Zhang, Y-L, Zhang, W-Y, Liu, X-Q, Wang, J-N, Zhang, X-L, Zhang, D-D, Jiang, $H-B$, Zhang, R. \& Sun, H-B Silver-coated rose petal green, facile, low-cost and sustainable fabrication of a SERS substrate with unique superhydrophobicity and high efficiency. Adv Opt Mater 1, 56-60 (2013).

9 Liu, Z., Yang, Z., Peng, B., Cao, C., Zhang, C., You, H., Xiong, Q., Li, Z. \& Fang, J. Highly sensitive, uniform, and reproducible surface-enhanced Raman spectroscopy from hollow Au-Ag alloy nanourchins. Adv Mater 26, 2431-2439 (2014).

10 Goldberg-Oppenheimer, P., Mahajan, S. \& Steiner, U. Hierarchical electrohydrodynamic structures for surface-enhanced Raman scattering. Adv Mater. 24 , OP175-OP180 OP174 (2012).

11 Jeon, T. Y., Park, S. G., Kim, D. H. \& Kim, S. H. Standing-wave-assisted creation of nanopillar arrays with vertically integrated nanogaps for SERS-active substrates. Adv Funct Mater 25, 4681-4688 (2015).

12 Coveney, P. V. \& Chin, J. Chirality and domain growth in the gyroid mesophase. Proc $R$ Soc A-Math Phys Eng Sci 462, 3575-3600 (2006).

13 Turner, M. D., Saba, M., Zhang, Q., Cumming, B. P., Schröder-Turk, G. E. \& Gu, M. Miniature chiral beamsplitter based on gyroid photonic crystals. Nat Photonics 7, 801-805 (2013).

14 Zhang, L., Lang, X., Hirata, A. \& Chen, M. Wrinkled nanoporous gold films with ultrahigh surface-enhanced Raman scattering enhancement. ACS Nano 5, 4407-4413 (2011).

15 Hsueh, H-Y, Chen, H-Y, Ling, Y-C, Huang, W-S, Hung, Y-C, Gwo, S. \& Ho, R-M Polymerbased SERS-active substrate with gyroid-structured gold multibranches. J Mater Chem C 2, 4667-4675 (2014).

16 Salvatore, S., Demetriadou, A., Vignolini, S., Oh, S. S., Wuestner, S., Yufa, N. A., Stefik, M., Wiesner, U., Baumberg, J. J., Hess, O. \& Steiner, U. Tunable 3D extended selfassembled gold metamaterials with enhanced light transmission. Adv Mater 25, 2713-2716 (2013)

17 Hsueh, H. Y., Chen, H. Y., Hung, Y. C., Ling, Y. C., Gwo, S. \& Ho, R. M. Well-defined multibranched gold with surface plasmon resonance in near-infrared region from seeding growth approach using gyroid block copolymer template. Adv Mater 25, 1780-1786 (2013)

18 Vignolini, S., Yufa, N. A., Cunha, P. S., Guldin, S., Rushkin, I., Stefik, M., Hur, K., Wiesner, U., Baumberg, J. J. \& Steiner, U. A 3D optical metamaterial made by selfassembly. Adv Mater 24, OP23-OP27 (2012).

19 Salvatore, S., Vignolini, S., Philpott, J., Stefik, M., Wiesner, U., Baumberg, J. J. \& Steiner, U. A high transmission wave-guide wire network made by self-assembly. Nanoscale 7, 1032-1036 (2014).

20 Tian, C., Deng, Y., Zhao, D. \& Fang, J. Plasmonic silver supercrystals with ultrasmall nanogaps for ultrasensitive SERS-based molecule detection. Adv Opt Mater 3, 404-411 (2015)

21 Tian, C., Li, J., Ma, C., Wang, P., Sun, X. \& Fang, J. An ordered mesoporous Ag superstructure synthesized via a template strategy for surface-enhanced Raman spectroscopy. Nanoscale 7, 12318-12324 (2015).

22 Tan, Y., Gu, J., Xu, L., Zang, X., Liu, D., Zhang, W., Liu, Q., Zhu, S., Su, H., Feng, C., Fan, G. \& Zhang, D. High-density hotspots engineered by naturally piled-up subwavelength structures in three-dimensional copper butterfly wing scales for surfaceenhanced Raman scattering detection. Adv Funct Mater 22, 1578-1585 (2012).

23 Merk, V., Kneipp, J. \& Leosson, K. Gap size reduction and increased SERS enhancement in lithographically patterned nanoparticle arrays by templated growth. Adv Opt Mater 1, 313-318 (2013).

24 Tan, Y., Gu, J., Zang, X., Xu, W., Shi, K., Xu, L. \& Zhang, D. Versatile fabrication of intact three-dimensional metallic butterfly wing scales with hierarchical sub-micrometer structures. Angew Chem Int Ed 50, 8307-8311 (2011).

25 Galusha, J. W., Richey, L. R., Gardner, J. S., Cha, J. N. \& Bartl, M. H. Discovery of a diamond-based photonic crystal structure in beetle scales. Phys Rev E 77, 050904 (2008).

26 Kirkensgaard, J. J., Evans, M. E., de Campo, L. \& Hyde, S. T. Hierarchical self-assembly of a striped gyroid formed by threaded chiral mesoscale networks. Proc Natl Acad Sci USA 111, 1271-1276 (2014) 
27 Thomas, E., Lescanec, R., Frank, F., Higgins, J., Klug, A. \& Hamley, I. Phase morphology in block copolymer systems (and discussion). Philos Trans $R$ Soc A-Math Phys Eng Sci 348, 149-166 (1994).

28 Michielsen, K. \& Stavenga, D. G. Gyroid cuticular structures in butterfly wing scales: biological photonic crystals. J R Soc Interface 5, 85-94 (2008).

29 Michielsen, K., De Raedt, H. \& Stavenga, D. G. Reflectivity of the gyroid biophotonic crystals in the ventral wing scales of the Green Hairstreak butterfly, Callophrys rubi. J $R$ Soc Interface 7, 765-771 (2010).

30 Winter, B., Butz, B., Dieker, C., Schröder-Turk, G. E., Mecke, K. \& Spiecker, E. Coexistence of both gyroid chiralities in individual butterfly wing scales of Callophrys rubi. Proc Natl Acad Sci USA 112, 12911-12916 (2015).

31 Schroder-Turk, G. E., Wickham, S., Averdunk, H., Brink, F., Fitz Gerald, J. D., Poladian, L., Large, M. C. \& Hyde, S. T. The chiral structure of porous chitin within the wingscales of Callophrys rubi. J Struct Biol 174, 290-295 (2011).

32 Saba, M., Thiel, M., Turner, M. D., Hyde, S. T., Gu, M., Grosse-Brauckmann, K. Neshev, D. N., Mecke, K. \& Schröder-Turk, G. E. Circular dichroism in biological photonic crystals and cubic chiral nets. Phys Rev Lett 106, 103902 (2011).

33 Saba, M., Wilts, B. D., Hielscher, J. \& Schröder-Turk, G. E. Absence of circular polarisation in reflections of butterfly wing scales with chiral Gyroid structure. Mater Today 1, 193-208 (2014).

34 Saranathan, V., Osuji, C. O., Mochrie, S. G., Noh, H., Narayanan, S., Sandy, A., Dufresne, E. R. \& Prum, R. O. Structure, function, and self-assembly of single network gyroid (14132) photonic crystals in butterfly wing scales. Proc Natl Acad Sci USA 107 11676-11681 (2010).

35 Ghiradella, H. Structure and development of iridescent butterfly scales: lattices and laminae. J Morphol 202, 69-88 (1989).

36 Zhu, W. \& Crozier, K. B. Quantum mechanical limit to plasmonic enhancement as observed by surface-enhanced Raman scattering. Nat Commun 5, 5228-5235 (2014).

37 Crozier, K. B., Zhu, W., Chu, Y., Wang, D., Banaee, M. in Frontiers of Surface-Enhanced Raman Scattering: Single Nanoparticles and Single Cells (eds Yukihiro Ozaki K. K. \& A Ricardo) 219-241 (John Wiley \& Sons, Ltd., USA, 2014).

38 Sau, T. K. \& Rogach, A. L. Nonspherical noble metal nanoparticles: colloid-chemical synthesis and morphology control. Adv Mater 22, 1781-1804 (2010).

39 Scherer, M. R. J. Synthesis and applications of double-gyroid-structured functional materials. (Doctor thesis, University of Cambridge, England, 2013).

40 Tan, Y., Gu, J., Xu, W., Chen, Z., Liu, D., Liu, Q. \& Zhang, D. Reduction of cuo butterfly wing scales generates Cu SERS substrates for DNA base detection. ACS Appl Mater Inter 5, 9878-9882 (2013).

41 Liu, Y., Yu, S., Feng, R., Bernard, A., Liu, Y., Zhang, Y., Duan, H., Shang, W., Tao, P. Song, C. \& Deng, T. A bioinspired, reusable, paper-based system for high-performance large-scale evaporation. Adv Mater 27, 2768-2774 (2015).

42 Sharma, B., Frontiera, R. R., Henry, A-I, Ringe, E. \& Van Duyne, R. P. SERS: materials, applications, and the future. Mat Today 15, 16-25 (2012).
43 Zhao, Y., Zhang, X-J, Ye, J., Chen, L-M, Lau, S-P, Zhang, W-J \& Lee, S-T Metallodielectric photonic crystals for surface-enhanced Raman scattering. ACS Nano $\mathbf{5}$ 3027-3033 (2011).

44 Urbas, A. M., Maldovan, M., DeRege, P. \& Thomas, E. L. Bicontinuous cubic block copolymer photonic crystals. Adv Mater 14, 1850-1853 (2002).

45 Takagi, H., Yamamoto, K. \& Okamoto, S. Ordered-bicontinuous-double-diamond structure in block copolymer/homopolymer blends. Europhys Lett 110, 48003 (2015).

46 Li, L., Szewczykowski, P., Clausen, L. D., Hansen, K. M., Jonsson, G. E. \& Ndoni, S. Ultrafiltration by gyroid nanoporous polymer membranes. J Membrane Sci 384, 126-135 (2011).

47 Cho, B-K, Jain, A., Gruner, S. \& Wiesner, U. Mesophase structure-mechanical and ionic transport correlations in extended amphiphilic dendrons. Science 305, 1598-1601 (2004).

48 Crossland, E. J., Kamperman, M., Nedelcu, M., Ducati, C., Wiesner, U., Smilgies, D-M Toombes, G. E., Hillmyer, M. A., Ludwigs, S. \& Steiner, U. A bicontinuous double gyroid hybrid solar cell. Nano Lett 9, 2807-2812 (2008).

49 Wei, D., Scherer, M. R., Bower, C., Andrew, P., Ryhänen, T. \& Steiner, U. A nanostructured electrochromic supercapacitor. Nano Lett 12, 1857-1862 (2012).

50 Kibsgaard, J., Jackson, A. \& Jaramillo, T. F. Mesoporous platinum nickel thin films with double gyroid morphology for the oxygen reduction reaction. Nano Energy 29, 243-348 (2016).

51 Torres-Rendon, J. G., Femmer, T., De Laporte, L., Tigges, T., Rahimi, K., Gremse, F., Zafarnia, S., Lederle, W., Ifuku, S. \& Wessling, M. Bioactive gyroid scaffolds formed by sacrificial templating of nanocellulose and nanochitin hydrogels as instructive platforms for biomimetic tissue engineering. Adv Mater 27, 2989-2995 (2015).

52 Asghar, K. A., Rowlands, D. A., Elliott, J. M. \& Squires, A. M. Predicting sizes of hexagonal and gyroid metal nanostructures from liquid crystal templating. ACS Nano 9 , 10970-10978 (2015)

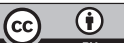

This work is licensed under a Creative Commons Attribution 4.0 International License. The images or other third party material in this article are included in the article's Creative Commons license, unless indicated otherwise in the credit line; if the material is not included under the Creative Commons license, users will need to obtain permission from the license holder to reproduce the material. To view a copy of this license, visit http:// creativecommons.org/licenses/by/4.0/

(C) The Author(s) 2018

Supplementary Information accompanies the paper on the NPG Asia Materials website (http://www.nature.com/am) 